\title{
Intracranial Meningeal Carcinomatosis from Prostate Cancer
}

\author{
Thalmann Sandrine ${ }^{1 *}$, Kuhn Peter ${ }^{1}$, Küng Marc ${ }^{2}$, Molteni Thierry ${ }^{3}$, Thoeny Harriet $C^{3}$ and Allal Abdelkarim S \\ ${ }^{1}$ Department of Radiation Oncology, HFR Fribourg, Switzerland
}

${ }^{2}$ Department of Medical Oncology, HFR Fribourg, Switzerland

${ }^{3}$ Department of Radiology HFR Fribourg, Switzerland

Submission: March 09, 2019; Published: April 10, 2019

*Corresponding author: Sandrine Thalmann, Department of Radiation Oncology, HFR Fribourg, Switzerland

\begin{abstract}
Due to an apparent increase in the number of cases of intracranial meningeal carcinomatosis in patients with prostate cancer observed in the early 2010s, we undertook a retrospective study to explore possible associated factors. Between 2008 and 2014 we identified 10 patients presenting with meningeal carcinomatosis. The time between the initial diagnosis, the first metastasis, the first chemotherapy, and the diagnosis of meningeal carcinomatosis were analyzed as well as the possible factors associated with this peculiar tumor spreading. The median time from the initial diagnosis to the development of meningeal carcinomatosis was 54 months. There were 21 months between the first chemotherapy and the diagnosis of meningeal carcinomatosis. Docetaxel was given in $80 \%$ of the patients. Along with the younger age of the patients (median 56 years) docetaxel appears to be the main common factor that characterized this cohort of patients. Several hypotheses that may explain this temporary increase of this metastasizing mode are elaborated and discussed in the present article.
\end{abstract}

Keywords: Prostate cancer; Meningeal carcinomatosis; Docetaxel

\section{Introduction}

In men prostate cancer is the most common cancer and the second most common cause of cancer death. The majority of patients, who develop metastases, will develop bone and / or lymph node metastases, whereas spreading to lungs, liver and brain is less common [1]. Meningeal carcinomatosis from prostate cancer has been described in some case reports or very small retrospective studies [2-10]. The diagnosis is made on contrast-enhanced CT and/or gadolinium-enhanced MRI. The cytological examination of the cerebrospinal fluid can also lead to the diagnosis although it is normal in 5 to $10 \%$ of cases. In the M.D. Anderson Cancer Center review of 16'280 patients with prostate cancer between 1944 and 1998, 131 patients had craniospinal metastases, 52 were diagnosed with CT or MRI and 78 postmortems; of these only 19 had intracranial meningeal carcinomatosis [11]. The present study was motivated by the unexpected increase of meningeal carcinomatosis cases from prostate cancer observed in our institution starting from 2012, with the aim to explore the associated factors.

\section{Materials and Methods}

Between 2003 and 2018 we identified 10 patients who presented with prostate cancer and intracranial meningeal carcinomatosis. All were observed between 2008 and 2014 and without associated brain metastasis except in two. The median age at the time of diagnosis of the prostate cancer was 56 years ( 49 to 79 ) and the average PSA was $331 \mathrm{ng} / \mathrm{ml}$ ( 5.5 to 2300 ). The diagnosis was made on prostate biopsies $(7 / 10)$, biopsy of an adenopathy, TURP or prostatectomy, respectively. The Gleason score was 7 or greater and perineural infiltration was found in five cases [13].

\section{Results}

The median time from the initial diagnosis of prostate cancer to the detection of the first bone metastasis was 21.5 months (0-95). Four patients were metastatic at initial diagnostic, three with only bone metastases and one with additional pulmonary and lymph node metastases. Four patients received pelvic radiotherapy, one patient underwent prostatectomy, four patients received an androgen deprivation therapy from the beginning and one patient refused any treatment. Chemotherapy was administrated to $80 \%$ of the patients between 15 to 101 months after the initial diagnosis (median 29 months). Only one patient didn't have chemotherapy and one received it after the detection of the meningeal carcinomatosis. The first line chemotherapy was based on docetaxel and then the second line on docetaxel again or cabazitaxel. Mitoxantrone was also 
used as subsequent line. The maximum chemotherapy lines administered was six.

Cerebral imaging was performed due to the appearance of one or a multiple symptom, including decreased visual acuity, diplopia, palpebral ptosis, facial hypoesthesia, vertigo, headache with or without vomiting, Horner's syndrome or hemiparesis. Meningeal carcinomatosis was diagnosed on CT alone [1], CT and MRI [6], or MRI alone [3]. The time between the initial diagnosis of prostate cancer and the development of meningeal carcinomatosis ranged from 7 to 127 months (median: 54 months), whereas this diagnosis was observed 10 to 48 months (median: 21 months) after the first chemotherapy and 8 to 64 months (median: 25.5 months) after the first occurrence of metastases elsewhere. Nine patients received whole-brain radiation therapy while the remaining patient died before the treatment had started. The time from the diagnosis of meningeal carcinomatosis to death ranges from 2 weeks to 29 months (median: 4 months). The median survival from the initial diagnosis was 58 months (26 to 129) and from the first metastasis 34 months (18 to 66 ).

\section{Discussion}

A meningeal carcinomatosis secondary to prostate cancer is rare. In 2012 fives cases were observed in our radiation oncology department. No such cases were found between 2003 and 2007 and after 2015 but ten cases were identified between 2008 and 2014, raising the question whether it was an isolated circumstance or a change in the disease evolution related to some specific factors. We considered several hypotheses, including age at the initial diagnosis, improved diagnostic techniques and the introduction of chemotherapy in the treatment of advanced prostate cancer.

\section{First hypothesis}

Our patients were 49 to 79 years old with a median age of 56 years at the time of the prostate cancer diagnosis which is quite below the 72 years (57 to 81 ) reported by Lin et al [9]. Would it be possible that younger patients may have more time to develop meningeal carcinomatosis? We found an interval of 7 months to more than 10 years from the initial diagnosis to the development of meningeal carcinomatosis. This is consistent with the publication from Nzokou A, et al. [12], who reported a range of 3 months to 7 years between the first diagnosis of prostate cancer and the detection of meningeal carcinomatosis in a series of patients with a higher median age (64.5 years), which doesn't support the age hypothesis.

\section{Second hypothesis}

Detection of meningeal carcinomatosis is more frequent nowadays. Seven of our patients underwent CT. Three examinations showed meningeal carcinomatosis and one showed bone metastases of the skull base with suspicion of meningeal invasion. Finally, nine patients had an MRI with pathological enhancement of the leptomeninges. This is consistent with the literature. Chamberlain et al [16] reports 30 vs $58 \%$ of false negative results for MRI vs CT. The use of MRI has thus become the gold standard for the search of meningeal lesions even though its sensitivity is below the repeated cerebrospinal fluid examination of $90 \%$ vs $76 \% 1,13$ as well as its specificity $75 \%$ vs $100 \% 13$. We didn't perform a cerebrospinal fluid examination in our patients. The use of MRI might have contributed to the higher detection of such metastases in this particular location.

\section{Third hypothesis}

Increased survival due to chemotherapy. Several randomized studies, including TAX 327 [14] and SWOG 9916 [15], show an increase in overall survival in patients with prostate cancer refractory to androgenic deprivation with docetaxel-based chemotherapy. The TAX 327 study showed a median survival of 19.2 months with docetaxel plus prednisone versus 16.3 months with mitoxantrone plus prednisone. The SWOG 9916 study also showed a median survival gain of 2 months and a time to progression gain of $50 \%$ (6.3 months vs 3.2 ). Docetaxel chemotherapy has therefore become a standard of care for the treatment of metastatic castration-resistant prostate cancers. Docetaxel was introduced in our hospital in 2005. In the present study, the median overall survival was of 32 months (18 to 66) since the first cycle of chemotherapy which is similar to the one reported by Caffo et al10. They found 31 cases of brain metastases, including 9 cases of meningeal carcinomatosis (incidence 3.3\% and $0.95 \%$ ) in a series of 943 patients with hormone-refractory prostate cancer who received chemotherapy based on Docetaxel. The incidence of meningeal carcinomatosis is therefore 3 times higher than the reported one $(0.3 \%)$ in the MD Anderson Cancer Center study $[12,16]$. Meningeal carcinomatosis was diagnosed before docetaxel treatment in 8 patients, during the treatment in 7 patients and after the treatment in 16 patients. In our study, only one patient was diagnosed before chemotherapy. The median post-meningeal carcinomatosis survival was 4 months [1-7], which is similar to our findings. An increase of meningeal carcinomatosis cases in solid tumors such as breast cancer (2$5 \%)$, lung cancer $(9-25 \%)$ and melanoma $(<23 \%)$ following the introduction of docetaxel-based chemotherapy 13 has also been described in the literature. The switch from docetaxel to abiraterone or enzalutamide as alternative treatment option beside docetaxel in the first-line treatment for castrationresistant prostate cancer in our hospital after 2014 coincidences with the decrease of newly diagnosed meningeal carcinomatosis. Our study has two main limitations. The first is the retrospective nature of the study including only 10 patients. The second is that we do not know the incidence of meningeal carcinomatosis before 2003 because autopsy is not systematically performed in our hospital and thus no meaningful comparison can be provided.

\section{Conclusion}

The apparent increase in the incidence of meningeal carcinomatosis was observed during the period where docetaxel 


\section{Open Access Journal of Neurology \& Neurosurgery}

was introduced as fist-line chemotherapy in metastatic castration-resistant prostate cancer patients. The increase of patients' survival due to docetaxel together with the inability of this drug to cross the blood-brain barrier may have led to a change in the recurrence patterns of this disease. Whether this was an incidental occurrence, or a real associated factor remains controversial and merits further investigations.

\section{References}

1. Orphaneos G, Ardavanis A (2010) Leptomeningeal metastases from prostate cancer: an emerging clinical conundrum. Clin Exp Metastasis 27(1): 19-23.

2. Weiner AB, Cortes-Mateus S, De Luis E, Duran I (2013) Dural metastases in advanced prostate cancer: a case report and review of the literature. Curr Urol 7(3): 166-168.

3. Cone LA, Fausel R, Gade-Andavolu R, Potts BE, Jennings LM, et al (2006) Leptomeningeal carcinomatosis in a patient with metastatic prostate cancer: Case report and literature review. Surgical neurology 65(4): 372-376.

4. Cante D, Franco P, Sciacero P, Girelli G, Casanova Borca V, et al. (2013) Leptomeningeal metastasis from prostate cancer. Tumori 99(1): $6 \mathrm{e}-10 \mathrm{e}$.

5. Honda M, Miyagawa I (2005) Prostatic meningeal carcinomatosis with low serum level of prostate-specific antigen. Urology 66(6): 1320.

6. Ramsis B (2002) Neurologic complications of prostate cancer. Am Fam Physician 65(9): 1834-1840.

7. Bernstein WB, Kemp JD, Kim GS, Johnson VV (2008) Diagnosing leptomeningeal carcinomatosis with negativ CSF cytology in advanced prostate cancer. JCO 26(19): 3281-3284.
8. Gupta R, Wang L, Wang J, Terry-Dettmer D, Sonpavde G (2012) Intracranial meningeal carcinomatosis in metastatic castration resistant prostate cancer: will extension of survival increase the incidence? Clinical Genitourinary Cancer 10 (4): 271-273.

9. Lin C, Turner S, Gurney H, Peduto A (2008) Increased detections of leptomeningeal presentations in men with hormone refractory prostate cancer: an effect of improved systemic therapy? J Med Imaging Radiat Oncol 52(4): 376-381.

10. Caffo O, Paglliarulo V, Prati V, Veccia A, Galligioni E, et al. (2012) Central nervous system metastases from castration-resistant prostate cancer in the docetaxel era. J Neurooncol 107(1): 191-196.

11. Tremont-Lukats IW, Bobustuc G, Lagos GK, Lolas K, kyritsis AP, et al. (2003) Brain metastases from prostate carcinoma: The M. D. Anderson Cancer Center experience. Cancer 98 (2): 363-368.

12. Nzokou A, Magro E, Guilbert F, Fournier JY, Bojanowski MW (2015) Subdural metastasis of prostate cancer 76(1): 123-127.

13. Straathof CSM, de Bruin HG, Dippel DWJ, Vecht CJ (1999) The diagnostic accuracy of magnetic resonance imaging and cerebrospinal fluid cytology in leptomeningeal metastasis. J Neurol 246(9): 810-814.

14. Berthold DR, Pond GR, Soban F (2008) Docetaxel plus prednisone or mitoxantrone plus prednisone for advanced prostate cancer: updated survival in the TAX 327 study. JCO 26 (2): 242-245.

15. Petrylak DP, Tangen CM, Hussain HA, Lara PN, Crawford ED, et al (2004) Docetaxel and Estramustine Compared with Mitoxantrone and Prednisone for Advanced Refractory Prostate Cancer. N Engl J Med 351(15): 1513-1520.

16. Chamberlin MC, Sandy AD, Press CA (1990) Leptomeningeal metastasis: a comparison of gadolinium enhanced MR and contrast-enhanced CT of the brain. Neurology 40(3): 435-438.

\section{Your next submission with Juniper Publishers will reach you the below assets}

- Quality Editorial service

- Swift Peer Review

- Reprints availability

- E-prints Service

- Manuscript Podcast for convenient understanding

- Global attainment for your research

- Manuscript accessibility in different formats

( Pdf, E-pub, Full Text, Audio)

- Unceasing customer service

Track the below URL for one-step submission

https://juniperpublishers.com/online-submission.php 